\title{
Solution of Skin-Effect Problems by means of the Hybrid SDBCI Method
}

\author{
G. Aiello, S. Alfonzetti, and N. Salerno \\ Dip. di Ingegneria Elettrica, Elettronica e Informatica, Università di Catania, \\ Viale A. Doria, 6, Catania, Italy
}

\begin{abstract}
This paper presents a singular version of the hybrid FEM-DBCI method to solve skin effect problems in open boundary domains. This version consists of overlapping the fictitious truncation boundary with the integration one, as in the FEM-BEM method.
\end{abstract}

Keywords-Finite elements, Boundary integrals, Hybrid methods, Skin effect

\section{INTRODUCTION}

Both FEM-BEM (Finite Element Method - Boundary Element Method) [1-2] and FEM-DBCI (Dirichlet Boundary Condition Iteration) [3-4] couple a differential equation, which governs the interior problem, with an integral one which makes use of the free-space Green function and expresses the unknown boundary condition on the fictitious truncation boundary.

In FEM-BEM the integration surface coincides with the truncation one, whereas in FEM-DBCI the integration surface is another surface strictly enclosed by the truncation boundary. In FEM-BEM the normal derivative of the potential on the truncation boundary is introduced and treated as an independent unknown, so that the interior problem is very often a pure Neumann one. In FEM-DBCI the normal derivative is not explicitly defined, but a numerical derivative is computed in the integral equation, so that the interior problem is a Dirichlet one.

A comparison was made in [5] between the two methods, concluding that FEM-BEM is more accurate than FEMDBCI, but requires more computing time:

This paper presents a singular version of the FEM-DBCI method in order to alleviate its major drawback, that is, the insertion of some element layers between the integration and truncation surfaces.

\section{FEM-SDBCI Formulation For SKIN EFFECT PROBLEMS}

Consider a system of z-directed straight conductors embedded in free space, as depicted in Fig. 1. The k-th conductor has magnetic reluctivity $v_{\mathrm{k}}$, electrical conductivity $\sigma_{\mathrm{k}}$ and cross sections $\mathrm{S}_{\mathrm{k}}$. The conductors carry a set of assigned source currents $\mathrm{I}_{\mathrm{k}}$, which are sinusoidal of angular frequency $\omega$ and globally balanced.

The z-directed magnetic vector potential $\mathrm{A}=\mathrm{A}(\mathrm{x}, \mathrm{y})$ satisfies the Laplace equation in free space, whereas in each conductor the Poisson equation holds:

$$
-v_{\mathrm{k}} \nabla^{2} \mathrm{~A}=\mathrm{J}
$$

where $J$ is the unknown current density, expressed as [6]:

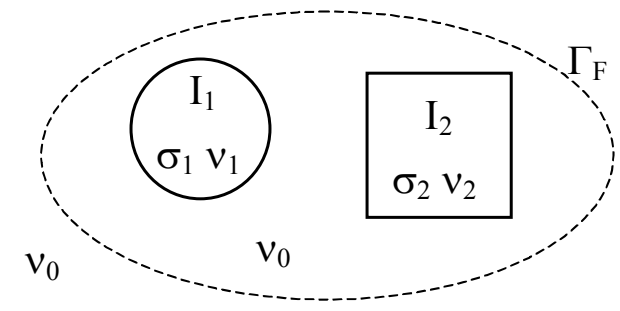

Fig. 1 - A set of conductors enclosed by the truncation boundary.

$$
J=\frac{I_{k}}{S_{k}}-j \omega \sigma_{k} A+j \omega \sigma_{k} \frac{1}{S_{k}} \int_{S_{k}} A d x d y
$$

Equation (1) is rewritten in integro-differential form as:

$$
-v_{\mathrm{k}} \nabla^{2} \mathrm{~A}+\mathrm{j} \omega \sigma_{\mathrm{k}}\left(\mathrm{A}-\frac{1}{\mathrm{~S}_{\mathrm{k}}} \int_{\mathrm{S}_{\mathrm{k}}} \mathrm{Adxdy}\right)=\frac{\mathrm{I}_{\mathrm{k}}}{\mathrm{S}_{\mathrm{k}}}
$$

The unbounded free space is truncated by means of a fictitious boundary $\Gamma_{\mathrm{F}}$ (see Fig. 1), on which an unknown Dirichlet condition is assumed to hold. Discretizing the obtained bounded domain by means of nodal finite elements of a given order, the following FEM system is derived:

$$
\mathbf{M A}=\mathbf{B}_{0}-\mathbf{M}_{\mathrm{F}} \mathbf{A}_{\mathrm{F}}
$$

where: $\mathbf{A}$ and $\mathbf{A}_{\mathrm{F}}$ are the vectors of the unknown values of the vector potential $\mathrm{A}$ in the nodes inside the domain and on the fictitious boundary $\Gamma_{\mathrm{F}}$, respectively, $\mathbf{M}, \mathbf{M}_{\mathrm{F}}$ are sparse matrices of geometrical coefficients, $\mathbf{B}_{0}$ is the part of the known term vector due to the source currents $I_{k}$.

Equation (4) alone is not sufficient to solve the problem because it only allows $\mathbf{A}$ to be obtained once the correct $\mathbf{A}_{\mathrm{F}}$ is known. If an incorrect guess is made for $\mathbf{A}_{\mathrm{F}}$, the resulting solution will be affected by a systematic error.

In order to solve the unbounded field problem, it is necessary to derive another equation relating the unknown vectors $\mathbf{A}$ and $\mathbf{A}_{\mathrm{F}}$. This equation is derived by

$$
\begin{aligned}
c_{i} A\left(P_{i}\right)+\int_{\Gamma_{F}} \mathrm{~A}(P) & \frac{\partial G\left(P, P_{i}\right)}{\partial n} d s= \\
& =A_{\text {ext }}\left(P_{i}\right)+\int_{\Gamma_{F}} v_{r} \frac{\partial A}{\partial n} G\left(P, P_{i}\right) d s
\end{aligned}
$$

where $c_{i}$ is the normalized angle of $D$ at node $P_{i}, A_{\text {ext }}$ is due to the external (lumped or distributed) source currents, the normal derivative is evaluated internally to $\mathrm{D}$, and $\mathrm{G}\left(\mathrm{P}, \mathrm{P}^{\prime}\right)$ is the free-space Green function, given by

$$
\mathrm{G}\left(\mathrm{P}, \mathrm{P}^{\prime}\right)=\frac{1}{2 \pi} \ln (1 / \mathrm{r})
$$


where $\mathrm{r}$ is the distance between $\mathrm{P}$ and $\mathrm{P}$ '. In numerical form, equation (5) reads

$$
\mathrm{c}_{\mathrm{i}} \mathrm{A}_{\mathrm{i}}+\sum_{\mathrm{k}} \sum_{\mathrm{m}} \mathrm{A}_{\mathrm{m}} \mathrm{h}_{\mathrm{im}}^{(\mathrm{k})}=\mathrm{A}_{\mathrm{ext}}\left(\mathrm{P}_{\mathrm{i}}\right)+\sum_{\mathrm{k}} \mathrm{v}_{\mathrm{r}}^{(\mathrm{k})} \sum_{\mathrm{m}} \mathrm{A}_{\mathrm{m}} \mathrm{g}_{\mathrm{im}}^{(\mathrm{k})}
$$

where: $h_{\mathrm{im}}^{(\mathrm{k})}=\int_{\mathrm{S}_{\mathrm{k}}} \alpha_{\mathrm{m}}(\mathrm{P}) \frac{\partial \mathrm{G}\left(\mathrm{P}, \mathrm{P}_{\mathrm{i}}\right)}{\partial \mathrm{n}} \mathrm{ds}$

$$
\mathrm{g}_{\mathrm{im}}^{(\mathrm{k})}=\int_{\mathrm{S}_{\mathrm{k}}} \frac{\partial \alpha_{\mathrm{m}}(\mathrm{P})}{\partial \mathrm{n}} \mathrm{G}\left(\mathrm{P}, \mathrm{P}_{\mathrm{i}}\right) \mathrm{ds}
$$

where $S_{k}$ is the side of the simplex finite element $E_{k}$ lying on $\Gamma_{\mathrm{F}}$; index $\mathrm{m}$ refers to the nodes of the element $\mathrm{E}_{\mathrm{k}}, \alpha_{\mathrm{m}}$ are the nodal shape functions, $v_{\mathrm{r}}^{(\mathrm{k})}$ is the relative permittivity of $\mathrm{E}_{\mathrm{k}}$. In matrix form we obtain:

$$
\mathbf{H} \mathbf{A}_{\mathrm{F}}=\mathbf{G A}+\mathbf{A}_{\text {ext }}
$$

where $\mathbf{H}$ and $\mathbf{G}$ are dense matrices. The coefficients $\mathrm{h}_{\mathrm{im}}$ are assembled in $\mathbf{H}$ if node $\mathrm{P}_{\mathrm{m}}$ belongs to to $\Gamma_{\mathrm{F}}$, otherwise they are assembled in $\mathbf{G}$ with a change of sign. On the contrary, the coefficients $g_{\text {im }}$ are assembled in $\mathbf{G}$ if node $P_{m}$ does not belong to to $\Gamma_{\mathrm{F}}$, otherwise they are assembled in $\mathbf{H}$ with a change of sign. Note that $\mathbf{H}$ is a square $\mathrm{N}_{\mathrm{F}} \times \mathrm{N}_{\mathrm{F}}$ matrix by construction, $\mathrm{N}_{\mathrm{F}}$ being the number of nodes lying on $\Gamma_{\mathrm{F}}$. Note also that null columns appear in $\mathbf{G}$ for the nodes of the elements not lying on $\Gamma_{\mathrm{F}}$; called $\mathrm{N}_{\mathrm{E}}$ the total number of nodes belonging to the elements lying on $\Gamma_{\mathrm{F}}$, the number of non null entries of $\mathbf{G}$ is $\mathrm{N}_{\mathrm{F}} \times\left(\mathrm{N}_{\mathrm{E}}-\mathrm{N}_{\mathrm{F}}\right)$. Since approximately $\mathrm{N}_{\mathrm{E}} \approx 2 \mathrm{~N}_{\mathrm{F}}$, the dense equation (9) requires a memory of about $2 \mathrm{~N}_{\mathrm{F}}^{2}$ memory locations, practically the same as FEM-BEM.

If node $\mathrm{P}_{\mathrm{i}}$ does not belong to $\mathrm{S}_{\mathrm{k}}$ the integrand functions in (7) and (8) are regular and a simple Gauss quadrature technique may be used [1]. If, on the contrary, the node $P_{i}$ belongs to $S_{k}$, the integrand functions are singular and the integrations are performed analytically: the coefficients $h_{i m}$ vanish, whereas the coefficients $g_{\text {im }}$ are calculated by means of analytical formulas.

\section{A NUMERICAL EXAMPLE}

In this section an example is given concerning a transmission line constituted by a coupled strip line on a ground plane, as depicted in Fig. 2. The geometrical data are: conductor width $\mathrm{w}=360 \mu \mathrm{m}$, conductor thickness $\mathrm{t}=36$, height from the ground plane $\mathrm{h}=100 \mu \mathrm{m}$, space between the conductors $\mathrm{s}=50 \mu \mathrm{m}$. The conductors are made of copper $\left(\sigma=5610^{6} \mathrm{~S} / \mathrm{m}\right)$, whereas the surrounding medium is assumed to have zero conductivity and vacuum permeability. Two analyses are performed in relation to common mode (CM) and differential mode (DM) source currents, whose frequency is set to $10 \mathrm{MHz}$. A rectangular truncation boundary is selected at a distance $\mathrm{d}=50 \mu \mathrm{m}$ from the conductors (see dashed line in Fig. 3). Due to symmetry reasons, the analyses are restricted to half the system, by imposing homogeneous Neumann and Dirichlet conditions on the symmetry axis (y-axis) for CM and DM, respectively,

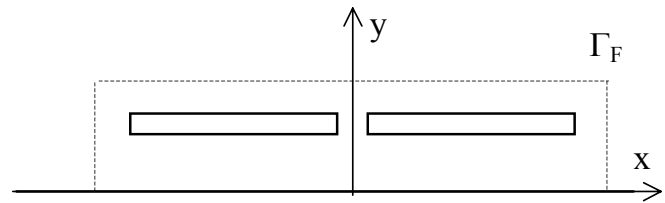

ground plane

Fig. 2 - Strip line on a ground plane and the relative truncation boundary.

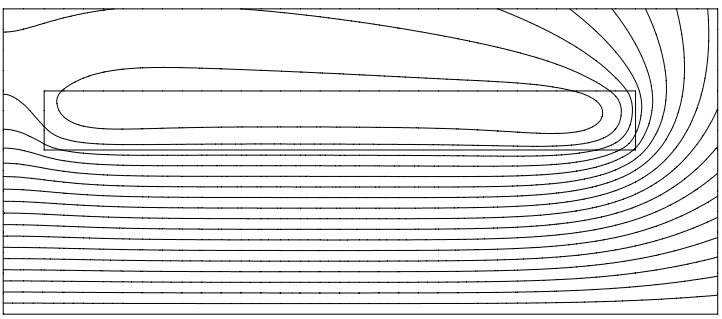

Fig. 3 - Contours of the magnitude of A for the CM case.

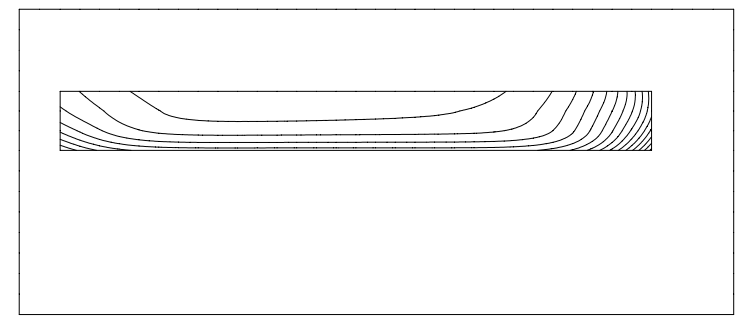

Fig. 4 - Contours of the magnitude of $\mathrm{J}$ for the $\mathrm{CM}$ case.

whereas in both cases homogeneous Dirichlet conditions hold on the ground plane (x-axis). The bounded domain is discretized by means of 1080 second-order triangles and 2263 nodes. Having set an end-iteration tolerance of $10^{-4}$ per cent, convergence is obtained with 17 and 19 GMRES iterations for CM and DM, respectively. The CPU times are $20.9 \mathrm{~s}$ and $20.5 \mathrm{~s}$ on a Pentium IV, 3.2 GHz, 3Gb RAM. Fig. 3 and 4 show the contours of the magnitude of the magnetic potential and of the current density, respectively, for the $\mathrm{CM}$ case.

\section{REFERENCES}

[1] P. P. Silvester and R. L. Ferrari, Finite Elements for electrical engineers, Cambridge University Press, Cambridge (U.K.), 1996.

[2] P. Bettess, "Infinite Elements," Int. J. Numer. Methods Eng., Vol. 11, pp. $53-64,1977$.

[3] G. Aiello, S. Alfonzetti, and S. Coco, "Charge Iteration: a Procedure for the Finite Element Computation of Unbounded Electrical Fields," Int. J. Numer. Methods Eng., vol. 37, pp. 4147-4166, December 1994.

[4] G. Aiello, S. Alfonzetti, S. Coco, and N. Salerno, "Finite Element Iterative Solution to Skin Effect Problems in Open Boundaries," Int. J. Numer. Modelling, special issue on 'Computational Magnetics', vol. 9, Jan.-April 1996, pp. 125-143.

[5] G. Aiello, S. Alfonzetti, G. Borzì, E. Dilettoso, N. Salerno, "Comparing FEM-BEM and FEM-DBCI for open-boundary electrostatic problems", European Phys. Journ,--Appl. Physics, vol. 39, pp 143-148, Aug. 2007.

[6] A. Konrad, "Integrodifferential finite element formulation of twodimensional steady-state skin effect problems," IEEE Trans. Magn., vol. 18, pp. 284-292, Jan. 1982. 